\title{
Homosexualités et fictions en France de 1981 à nos jours, É. Bordas et O. Heathcote (dir.)
}

\section{Stefano Genetti}

\section{(2) OpenEdition}

1 Journals

\section{Edizione digitale}

URL: http://journals.openedition.org/studifrancesi/10602

DOI: 10.4000/studifrancesi. 10602

ISSN: 2421-5856

\section{Editore}

Rosenberg \& Sellier

\section{Edizione cartacea}

Data di pubblicazione: 1 décembre 2017

Paginazione: 586-587

ISSN: 0039-2944

\section{Notizia bibliografica digitale}

Stefano Genetti, «Homosexualités et fictions en France de 1981 à nos jours, É. Bordas et O. Heathcote (dir.)», Studi Francesi [Online], 183 (LXI | III) | 2017, online dal 01 février 2018, consultato il 21 janvier 2021. URL: http://journals.openedition.org/studifrancesi/10602 ; DOI: https://doi.org/10.4000/ studifrancesi. 10602

Questo documento è stato generato automaticamente il 21 janvier 2021.

\section{(c) (1)}

Studi Francesi è distribuita con Licenza Creative Commons Attribuzione - Non commerciale - Non opere derivate 4.0 Internazionale. 


\title{
Homosexualités et fictions en France de 1981 à nos jours, É. Bordas et $\mathrm{O}$. Heathcote (dir.)
}

\author{
Stefano Genetti
}

\section{NOTIZIA}

Homosexualités et fictions en France de 1981 à nos jours, dirigé par Éric BORDAS et Owen HEATHCOTE, «Revue critique de fixxion française contemporaine» 12, 2016, 229 pp.

1 Lanciata da Pierre Schoentjes nel 2010, la «Revue critique de fixxion française contemporaine» si è imposta come uno dei più stimolanti periodici on-line di argomento contemporaneo. Sensibile alle interazioni del letterario con altre arti e discipline, nonché alle attuali tendenze della critica quali Cultural Studies e écopoétique, questa rivista bilingue francese/inglese è organizzata in numeri tematici la cui curatela è delegata a specialisti internazionali. Nel caso in questione ci si interroga sull'omosessualità come esperienza di finzione e come spazio di elaborazione, contestazione e contaminazione di discorsi sulle omosessualità in connessione con $\mathrm{i}$ radicali mutamenti che caratterizzano l'arco temporale preso in esame, dalla depenalizzazione dell'omosessualità, poco prima dell'irruzione dell'Aids, fino al mariage pour tous, dalla repressione alla gestione sociale delle minoranze sessuali e dalle valenze politiche che assume il costituirsi di una storia culturale Lgbt al destabilizzante ripensamento queer delle identità di genere (Présentation, pp. 1-4).

2 Tra i contributi riguardanti le condizioni di produzione, diffusione e ricezione della letteratura gay e lesbica, Nicholas GIGUère e Luc P INHAS offrono una rassegna comparativa intitolata Presse gaie, littérature et reconnaissance homosexuelle au tournant des années 1980 en France et au Québec: "Gai Pied», "Masques», les éditions Persona et "Le Berdache» (pp. 5-17), mentre Jérémy LAMBERT porta ad esempio le Éditions gaies et lesbiennes inaugurate nel 1997, la cui collana «Le bonheur est à tout le monde» 
restituisce una visione "naturalizzata" e normalizzante dei rapporti omosessuali (Le roman sentimental gay francophone, pp.18-26). Sugli effetti spesso paradossali della censura si sofferma O. HEATHCOTE in Banning the Boy. Homosex, Censorship and the Work of Éric Jourdan (pp. 27-33), figlio adottivo di Julien Green, collaboratore della rivista homophile «Arcadie» e autore, tra l'altro, del romanzo Les mauvais anges, autocensurato in fase di redazione e di revisione e ciò nonostante vietato sia nel 1956 che nel 1974. Se in questo caso la censura consolida un'identità volutamente marginale, in quello di Violette Leduc essa ha snaturato una pratica letteraria la cui portata rivoluzionaria risiede nel rifiuto di scindere l'eros "clandestino" dall'esistenziale e dal sentimentale, come sottolinea Anaïs FRANTZ DE SPOT nel ripercorrere la genesi e la vicenda editoriale di Ravages (Pourquoi lire "Thérèse et Isabelle" aujourd'hui?, pp. 224-229, nella sezione «ReLire»).

3 Vari articoli sono dedicati all'ambivalente visibilità che agli omosessuali ha conferito la tragica eppure fondante cesura rappresentata dall'Aids. In Dreuilhe's "Corps à corps": Metaphor/Phantasy and Mobilisation (pp. 96-109), Jean-Pierre B OULÉ torna sull'immaginario bellico derivato da Susan Sontag che, in questo anomalo «Journal de Sida» scritto in francese a New York, si rivela al contempo strategia di sopravvivenza e appello alla militanza comunitaria. Sulla scia di Bachtin, Derrida e Judith Butler, Benjamin GAGNON C HAINEY studia la disorientante erotizzazione della relazione terapeutica nel secondo volet della cosiddetta trilogie du sida di Guibert, dove al dottor Chandi subentra il personaggio di Claudette Dumouchel (Du protocole compassionnel à l'anarchie passionnelle: le vacillement interdiscursif des orientations sexuelles. Vers l'espoir d'une médecine au féminin, pp. 84-95). Contestazione del sapere medico e dei fondamenti biologici dell'identità di genere sono al centro dell'intervento di Jason HARTFORD, Towards a Queer Ecology. Science and Nature in "Un ruban noir" (Vincent Borel, 1995) (pp. 34-43), un romanzo dove il narratore e protagonista è un infermiere sieropositivo presso un ospedale parigino nel 1988. Dal canto suo, Ârash AMINIAN TABRIZI analizza, nel contesto del dibattito sul "sesso sicuro", le implicazioni sovversive dell'auto-pornobiografia nel terzo romanzo di Dustan (Sur/vivre aux limites (1): sexistence et transgression dans "Plus fort que moi" de Guillaume Dustan, pp. 44-59).

4 Se alle controversie sollevate dal romanzo e dal film Les nuits fauves di Cyril Collard fa letteralmente da controcanto l'estetica camp del film musicale degli attivisti O. Ducastel e J. Martineau commentato da Renaud LAGABRIELLE in Représentations (dés)enchantées: "Jeanne et le garçon formidable" (pp. 153-163), la dimensione vitalistica del desiderio omosessuale viene valorizzata, tra relazionalità alternativa (Leo Bersani) e jouissance (Lacan), da Enda MCCAFFREY nel suo (Im)personal Relationality in Alain Guiraudie's "Ici commence la nuit" (pp. 60-71) a proposito del romanzo pubblicato nel 2014, l'anno dopo il film L'inconnu du lac dello stesso Guiraudie, nel cui cinema Olivier CHEVAL vede l'espressione di un'etica anarchica e di un egualitarismo erotico (Voici venu le temps d'aimer, les camaraderies utopiques d'Alain Guiraudie, pp.164-173), mentre Jacques DÜRRENMATT sottolinea il carattere pioneristico, nel 1996, della prima bande dessinée dichiaratamente gay (Une question d'ego: le "Journal" de F. Neaud, pp. 174-184).

5 A due universi socio-culturali opposti sono riconducibili le tematiche familiari affrontate da Robert PAYNE nell'articolo Lesbianism and Maternal Ambivalence in Hélène de Monferrand's "Les amies d'Héloïse" and "Les enfants d'Héloïse" (pp. 120-129) e da Maxime FOERSTER in Du «Familles, je vous hais!» au transfuge de classe: le cas Eddy Bellegueule (pp. 71-83). Nell'uno, la decostruzione di una visione normativa e istituzionalizzata della 
maternità viene discussa in merito al romanzo epistolare di ambientazione aristocratica, premio Goncourt nel 1990, e alla sua continuazione del 1997, dove è invece centrale il punto di vista dei figli, compresa la lesbica Mélanie. Nell'altro, dominazione eterosessista e di classe si intrecciano nel "divenire autore" di Édouard Louis con il best seller En finir avec Eddy Bellegueule, auto-socio-biografia della honte sulla scia di Bourdieu e di Éribon.

In Homosexualité vs. homo-sexualité chez Marguerite Duras (pp. 143-152), Laurent CAMERINI cerca di superare ogni binarismo nel suo interrogare le prese di posizione omofobe dell'autrice a partire dall'incontro con Yann Lemée che, in virtù di una progressiva mise en fiction che sovrappone identità omosessuale e ebraica, diventa nell'opera Yann Andréa Steiner. Su due figure fondamentali nella costruzione di una memoria culturale e letteraria Lgbtq si soffermano Eva FEOLE e Christina MARCANDIER. La prima rinviene le tracce dell'eredità di M. Wittig in un romanzo del 1986 dove A. Garréta rende indeterminabile il genere dell'io narrante e dell'amante: mediante l'inscrizione dell'eros e della violenza nel corpo testuale, la definizione-classificazione insita nella nozione stessa di genere viene denunciata in quanto strumento di oppressione rispetto al quale la scrittura svolge una funzione liberatoria (Le déchaînement littéraire: "Sphinx" d'Anne Garréta et "Le corps lesbien" de Monique Wittig, pp. 110-119). La seconda si aggira invece tra le proiezioni romanzesche del fantasma di Foucault, personaggio e archivio di scenari, idee, atteggiamenti in libri, tra gli altri, di Hervé Guibert, Tristan Garcia, Mathieu Lindon e Laurent Binet (Le corps-texte de Michel Foucault, personnage romanesque et énoncé fictionnel, pp.130-142). Il silenzio del pensatore riguardo all'Aids, da molti ritenuto all'epoca colpevole, è reinterpretato alla luce della tensione tra coraggio della verità e mentir vrai che attraversa la sua opera, come rilevato da François Noudelmann in Le génie du mensonge (Paris, Max Milo, 2015).

7 A completamento di questo numero figurano interessanti incontri e testimonianze. Renaud CHANTRAINE intervista l'infaticabile animatore di iniziative culturali Patrick Cardon: quarante ans de militantisme culturel Lgbt (pp. 185-196): dal gruppo di riflessione su sessualità e politica Sexpol al centro di cultura camp L'Éventail, attraversando il catalogo delle edizioni GayKitschCamp e rievocando le rassegne cinematografiche su questioni di genere, ad esempio in merito all'adattamento cinematografico di Les amitiés particulières realizzato da Delannoy nel 1964, vent'anni dopo la pubblicazione del romanzo di Peyrefitte che, nella rubrica «Re-Lire», Jean-Christophe CAVALLIN rivisita in quanto storia proto-queer di amicizie défendues - allo stesso tempo proibite e difese dove la sessualità rimane sullo sfondo (Des enfants d'un naturel remarquable. Notes de lecture sur "Les amitiés particulières" de Roger Peyrefitte, pp. 213-223). Sessualità e interculturalità si intrecciano anche nella conversazione di Antoine IDIER con Abellah Taïa («Sortir de la peur», Construire une identité homosexuelle arabe dans le monde postcolonial. Entretien avec Abdellah Taïa, pp.197-207). Anche nel suo recentissimo romanzo epistolare a ritroso Celui qui est digne d'être aimé, lo scrittore e regista marocchino narrativizza il complesso di inferiorità linguistico-culturale tra venerazione e sottomissione, tra tradimento e emancipazione, tra tradizione araba e modelli francesi. Nella sezione «Carte blanche», infine, Mathieu RIBOULET rivendica la necessità di «écrire sur le désir des hommes pour les hommes» (p. 210) declinato nei suoi versanti luminosi oppure oscuri e accidentati, anche in risposta alle inoffensive e tuttavia eloquenti critiche "omofobe" subite dall'autore di L'amant des morts e Lisières du corps (Le sexe, le désir, le texte, pp. 209-212). Dal canto suo, Chantal CHAWAF insiste sulla 
ricerca di una «langue homopoétique de la matérialité», atta a rendere conto della relazione profondamente omosessuale tra donna e vita (Homosexualité de la Vie, p. 208). 